\title{
Effect of TERT on the growth of fibrosarcoma via caspase-3, survivin and PKB
}

\author{
QIUYE MA ${ }^{1}$, YIDONG YU ${ }^{1}$, LINLIN DAI ${ }^{2}$, XUEHUA QU ${ }^{2}$, SHAN CONG $^{2}$ and HONGSUO LIANG ${ }^{3}$ \\ ${ }^{1}$ Department of Orthopedics, Chinese Medicine Hospital of Jiulongpo, Chongqing 400080; ${ }^{2}$ Department of Orthopedics, \\ The Fourth Affiliated Hospital of Harbin Medical University, Harbin, Helongjiang 150001; ${ }^{3}$ Department of \\ Orthopedics, Nanning Second People's Hospital, Nanning, Guangxi 530031, P.R. China
}

Received October 19, 2015; Accepted January 6, 2017

DOI: $10.3892 / \mathrm{ol} .2017 .6373$

\begin{abstract}
The present study explored the effect of telomerase reverse transcriptase (TERT) on the growth and apoptosis of fibrosarcoma, and investigated the potential molecular signalling pathways underlying its effect. A plasmid was constructed in order to overexpress TERT and siRNA was used to knockdown TERT. The effect of TERT on fibrosarcoma cells in vitro was studied by performing reverse transcription-quantitative PCR and western blotting to determine the expression of p53, survivin, caspase-3, caspase-7 and PKB. Knockdown of TERT suppressed cell growth, decreased fibrosarcoma volume, decreased survivin and PKB expression, and increased caspase-3 expression. The results of the present study suggest that TERT regulates the growth of fibrosarcoma in vitro and in vivo, and that this is associated with the expression of caspase-3 and survivin, in addition to the PKB signalling pathway.
\end{abstract}

\section{Introduction}

Telomerase reverse transcriptase (TERT), known as hTERT in humans, is the catalytic component of telomerase, a type of nuclear reverse transcriptase responsible for telomere extension in cells (1). A previous study demonstrated that abnormal expression of hTERT leads to the progressive shortening of telomeres, which could induce cell immortalization and malignant tumor growth (2). The function of hTERT in vivo and in vitro was studied directly by cloning the open reading frame (ORF) of the target gene into expression vectors with different protein tags, a technique used in a previous gene function study (3).

In the current study, ORF expression cloning technology was used with the TERT gene as a target to design and construct

Correspondence to: Dr Hongsuo Liang, Department of Orthopedics, Nanning Second People's Hospital, 13 Dancunlu, Nanning, Guangxi 530031, P.R. China

E-mail: hongsuoliang@126.com

Key words: telomerase reverse transcriptase, fibrosarcoma, RNA interference, apoptosis a TERT-ORF clone vector and produce virus particles with the ability to express TERT through viral packaging. The effects of TERT on fibrosarcoma in vitro were studied.

\section{Materials and methods}

Cell lines and cell culture. Human fibrosarcoma HT1080 cells were purchased from the American Type Culture Collection (Manassas, VA, USA) and were cultured in Minimal Essential Medium supplemented with $10 \%$ fetal bovine serum (both from Gibco; Thermo Fisher Scientific, Inc., Waltham, MA, USA), $100 \mathrm{U} / \mathrm{ml}$ penicillin and $100 \mu \mathrm{g} / \mathrm{ml}$ streptomycin at $37^{\circ} \mathrm{C}$ with $5 \% \mathrm{CO}_{2}$. Lenti-Pac 293Ta cells (GeneCopoeia, Inc., Rockville, MY, USA) cultured using Dulbecco's modified Eagle's medium supplemented with $10 \%$ fetal bovine serum and penicillin/streptomycin in the same conditions. Human non-small cell lung carcinoma cell line H1299 cells (American Type Culture Collection) were cultured with RPMI-1640 supplemented with $10 \%$ fetal bovine serum and penicillin/streptomycin, also in the same conditions.

Transduction of the Lv130 human immunodeficiency virus (HIV) lentiviral vector. A total of $10 \mathrm{ng}$ of Lv130 HIV lentiviral vector (GeneCopoeia China Inc., Guangzhou, China) was added to $100 \mu \mathrm{l}$ competent Escherichia coli cells (cat no., C7373-03; Invitrogen; Thermo Fisher Scientific, Inc.) and incubated on ice for $30 \mathrm{~min}$, then immediately transferred to a $42^{\circ} \mathrm{C}$ water bath and heat shocked for $60 \mathrm{sec}$, then transferred to ice for $2 \mathrm{~min}$. Super optimal culture medium (200 $\mu$ l; Sigma-Aldrich China, Beijing, China) was added and the sample was incubated at $37^{\circ} \mathrm{C}$ with agitation for $1 \mathrm{~h}$ at $200 \mathrm{rev} / \mathrm{min}$, then $100 \mu \mathrm{l}$ was cultured on an LB plate with ampicillin at $37^{\circ} \mathrm{C}$ overnight. The plasmid was isolated with a Plasmid Miniprep kit (cat. no., PFM250; Sigma-Aldrich China) according to the manufacturer's protocol. Restriction endonuclease digestion and $1 \%$ agarose gel electrophoresis with ethidium bromide were performed to confirm that the TERT sequence had been incorporated into the plasmid.

Construction of the recombinant plasmid with lentivirus vector and TERT gene DNA. The TERT gene was obtained by polymerase chain reaction (PCR) analysis. The total RNA of nude mice brain (supplied by Laboratory Animal Center of the 
Harbin Medical University, Harbin, China) was isolated with RNeasy Plus Universal kits [cat. no. 73404; QIAGEN (Suzhou) Translational Medicine Co., Ltd., Suzhou, China] according to the manufacturer's protocol. Primers were designed according to the mouse TERT gene sequences in GenBank. Primer sequences for TERT were as follows: Forward, 5'-GCGGTA GGCGTGTACGGT-3' and reverse, 5'-CGATCTCGAACT CGTGGC-3'. Primers contained restriction sites for NheI and $B s p 119 I$. Reverse transcription (RT)-PCR was performed with a One Step RT-PCR kit [cat. no. 210212; QIAGEN (Suzhou) Translational Medicine Co., Ltd.]. The RT-PCR product (TERT gene) and the lentiviral vector were digested with NheI and Bsp119I restriction endonucleases. The products of enzyme digestion were purified with a QIAquick Gel Extraction kit [cat. no. 28704; QIAGEN (Suzhou) Translational Medicine Co., Ltd.] and ligated with T4 DNA ligase to construct the recombinant plasmid.

Packaging of the virus. Cells at a confluence of $70-80 \%$ were used for transfection. The lentiviral packaging plasmid mixture (including $0.5 \mu \mathrm{g}$ recombinant plasmid and $1 \mu \mathrm{l}$ virus) was co-transfected into 293Ta cells and H1299 cells, which were incubated at room temperature for $25 \mathrm{~min}$, then added into 6-well plates and cultured at $37^{\circ} \mathrm{C}$ with $5 \% \mathrm{CO}_{2}$. The medium containing transfection mixture residues was discarded and fresh medium was added following $12 \mathrm{~h}$ incubation. The cell supernatant was collected after $48 \mathrm{~h}$ by centrifugation $\left(1,000 \mathrm{x}\right.$ g at $4^{\circ} \mathrm{C}$ for $\left.5 \mathrm{~min}\right)$, then a $0.45 \mu \mathrm{m}$ polyvinylidene fluoride (PVDF) film was used to filter and harvest the packaged virus particles.

Overexpression of TERT. The recombinant lentiviral overexpression vector was transfected into HT1080 cells using Lipofectamine 2000 (cat. no. 11668027; Thermo Fisher Scientific Inc.), according to the manufacturer's protocol.

RNA interference (RNAi) of TERT. HT1080 cells at confluence of $70-80 \%$ were used for RNAi. TERT-siRNA: AATCAGACAGCACTTGAAGAGGG was used for transfection of HT1080 cells using Lipofectamine 2000. Fluorescent images were captured following culture for $48 \mathrm{~h}$ to observe the growth status of the cells. Five images of each culture well were captured in the visual fields located in the center, upper, lower, left and right of the well.

RT-quantitative (q) PCR. Total RNA was extracted from cells subjected to the knockdown or overexpression of TERT after $72 \mathrm{~h}$, using the RNeasy Mini kit (Qiagen, Inc., Valencia, CA, USA) according to the manufacturer's protocol. Total RNA $(1 \mu \mathrm{g})$ was subjected to reverse transcription using a reverse transcription system (cat. no. A3500; Promega Corporation, Madison, WI, USA). qPCR was performed using SYBR-Green PCR Master Mix (Promega Corporation). Thermocycler conditions were as follows: $95^{\circ} \mathrm{C}$ for $45 \mathrm{sec} ; 95^{\circ} \mathrm{C}$ for $5 \mathrm{sec}$; and $60^{\circ} \mathrm{C}$ for $30 \mathrm{sec}$, for 40 cycles. Primers used were as follows: p53 forward, 5'-GCCATGGCCATCTACAAG-3' and reverse, 5'-CCTTCCACCCGGATA AGAT-3'; survivin forward, 5'-TTCAAGAACTGGCCCTTC-3' and reverse, 5'-CCTTAA AGCAGAAAAAACACTG-3'; caspase-3 forward, 5'-TTC TTCAGAGGCGACTACT-3' and reverse, 5'-TCCCACTGT

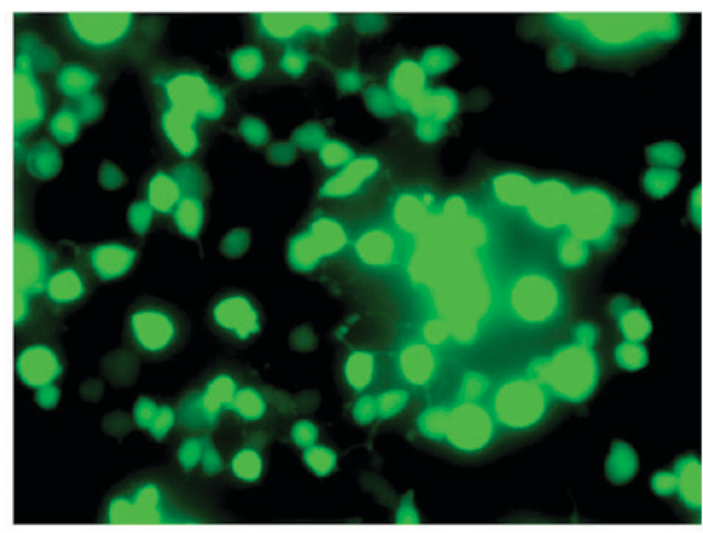

Figure 1. Fluorescence microscopy image of HT1080 cells following transfection with the human telomerase reverse transcriptase plasmid (magnification, x200).

CTGTCTCAAT-3'; and caspase-7 forward, 5'-TCTTTGCTT ACTCCACGGTT-3' and reverse, 5'-ACCCTGGTCAGGATC TGCAT-3'; GAPDH (internal control) forward, 5'-TGTGGG CATCAATGGATTTGG-3' and reverse, 5'-ACACCATGT ATTCCGGGTCAAT-3'. The results were quantified using the $2^{-\Delta \Delta C q} \operatorname{method}(4)$.

Detection of the expression of protein kinase $B(P K B)$ with western blotting. Briefly, cells that had been subjected to the knockdown or overexpression of TERT were lysed $72 \mathrm{~h}$ after RNAi in lysis buffer containing $50 \mathrm{mM}$ Tris (pH 7.4), $150 \mathrm{mM} \mathrm{NaCl}, 1 \%$ Triton X-100, $1 \%$ sodium deoxycholate, $0.1 \%$ SDS, $1 \mathrm{mM}$ EDTA and protease inhibitor at $4^{\circ} \mathrm{C}$ for $2 \mathrm{~h}$. Total protein $(50 \mu \mathrm{g})$ was separated by $15 \%$ SDS-PAGE and transferred to a PVDF membrane. The membrane was incubated with primary antibodies against PKB (dilution, 1:1,000; United States Biological, Salem, MA, USA) at $4^{\circ} \mathrm{C}$ overnight. The secondary antibodies were added at a dilution of 1:1,000, incubated at RT for $2 \mathrm{~h}$ and the bands were stained with DAB. They were quantified using $\beta$-actin (dilution, 1:1,000; antibody cat. no. 143128; United States Biological) as the control. The protein bands were quantified using Image $\mathbf{J}$ software (version k 1.45; National Institutes of Health, Bethesda, MA, USA; https://imagej.en.softonic.com/).

Statistical analysis. Statistical analysis was performed using the SPSS software (version 17.0; SPSS, Inc., Chicago, IL, USA) and a one-way analysis of variance was conducted for the comparison between groups. $\mathrm{P}<0.05$ was considered to indicate a statistically significant difference.

\section{Results}

Successful construction of the recombinant plasmid with the lentivirus vector and TERT gene DNA. The recombinant plasmid was isolated and confirmed to possess the ORF of the TERT gene by enzymatic digestion with restriction endonucleases NheI and Bsp119I, and sequencing of the plasmid (data not shown). Green fluorescence was observed in viable HT1080 cells following transfection with the recombinant TERT plasmid (Fig. 1), confirming expression of the plasmid. 
A

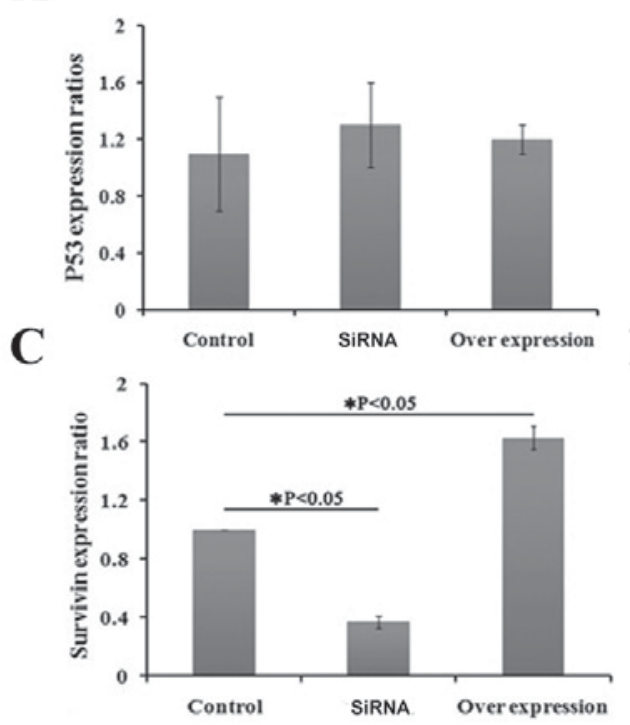

B
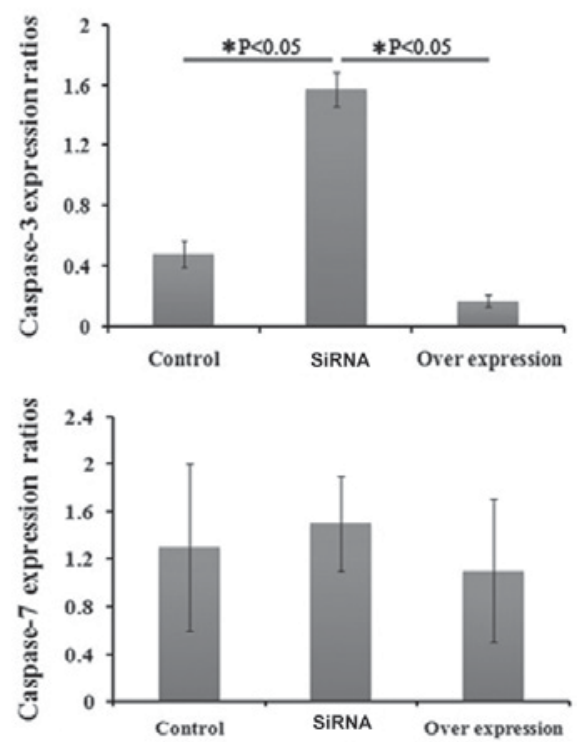

Figure 2. Reverse transcription-quantitative polymerase chain reaction analysis of the expression of (A) p53, (B) caspase-3, (C) survivin and (D) caspase-7. Error bars represent standard deviation. siRNA, small interfering RNA.
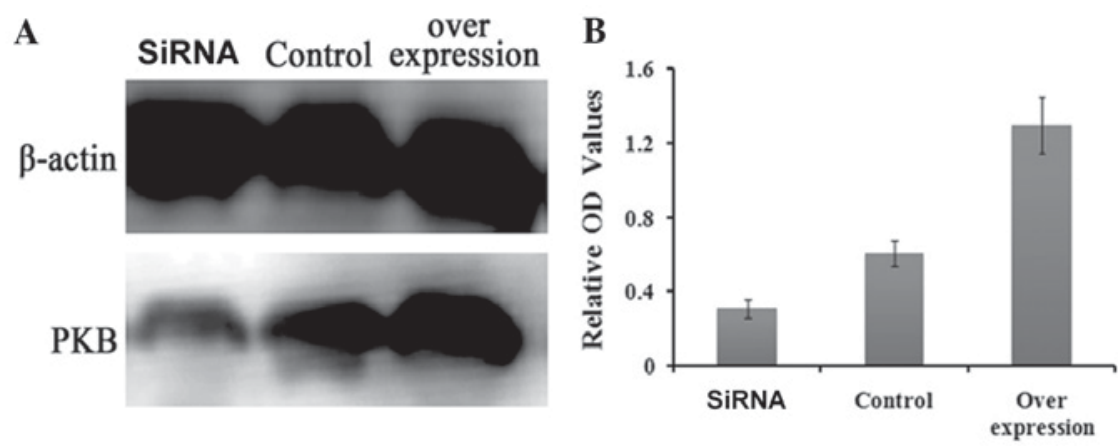

Figure 3. Western blotting results. (A) Western blot showing PKB expression in control, TERT siRNA-treated and TERT plasmid-treated HT1080 cells. (B) Quantification of PKB protein levels from western blotting. Error bars represent standard deviation. TERT, telomerase reverse transcriptase; PKB, protein kinase B; OD, optical density; siRNA, small interfering RNA.

Survivin and PKB expression decreases but caspase-3 expression increases following TERT knockdown. The effects of recombinant plasmid transfection on the expression levels of p53, survivin, caspase-3 and caspase-7 in HT1080 cells were detected by RT-qPCR. There was no significant difference in the expression levels of p53 and caspase-7 genes prior to and following TERT knockdown ( $\mathrm{P}>0.05$; Fig. 2). However, the expression levels of survivin decreased significantly and the expression levels of caspase-3 increased significantly following TERT knockdown $(\mathrm{P}<0.05$; Fig. 2). The expression levels of survivin increased significantly and the expression levels of caspase-3 decreased significantly following TERT overexpression (Fig. 2).

Western blotting analysis revealed that the expression of PKB decreased significantly at the protein level following TERT knockdown $(\mathrm{P}<0.05)$, while it increased significantly when TERT was overexpressed $(\mathrm{P}<0.05$; Fig. 3$)$.

\section{Discussion}

The biological role of TERT is to add telomeric DNA to the end of eukaryotic chromosomal DNA to ensure the activity of cells (5). Telomeres serve an important role in maintaining cellular chromosome stability and activity in the cells of various species (6). In the present study, TERT overexpression significantly promoted the growth of HT1080 cells, while TERT knockdown significantly inhibited growth. TERT affects cell growth, as it regulates the expression of telomerase, which affects telomere length, and the length of telomeres can affect the cell growth state (7-9). Previous studies have demonstrated that TERT knockdown inhibits the growth of bladder cancer and prostate cancer $(2,10)$. These results suggest that the TERT gene can control fibrosarcoma growth.

Previous studies have identified that TERT inhibits cell apoptosis $(11,12)$, which could account for the increased tumor growth observed following TERT overexpression. In the current study, the expression levels of caspase-3, caspase-7, survivin and p53 were measured prior to and following TERT knockdown. TERT knockdown did not significantly affect the expression of caspase-7 or p53; however, it significantly affected the expression of caspase-3 and survivin. Survivin has been demonstrated to promote cell survival (13), whereas caspase-3 promotes apoptosis (14). In the present study, TERT 
overexpression and knockdown significantly decreased and increased caspase-3 expression, respectively, indicating that TERT may serve a role in the growth of fibrosarcoma through caspase-3 and survivin.

PKB is a signalling molecule that regulates cell apoptosis and survival, and serves an important role in wound repair $(15,16)$. In the present study, TERT was identified to regulate the expression of PKB. Previous studies have demonstrated that the PKB signaling pathway is widely associated with cell apoptosis, survival, growth and protein synthesis (17-20). Signal transmission in the cytoplasm through PKB promotes cell cycle progression by glycogen synthase kinase-3 $\beta$ (21), and accelerates cell apoptosis through the inhibition of p21 or p53 (22). The results of the present study suggest that TERT affects the growth of fibrosarcoma via cell survival and apoptosis through the PKB signalling pathway.

The present study established a fibrosarcoma cell model overexpressing TERT. In this cell line, the effects of TERT on cell growth appeared to be mediated through the survivin, caspase-3 and PKB signalling pathways. These results provide important information for the application of hTERT-targeted therapies for the treatment of fibrosarcoma and similar malignancies, such as giant cell bone tumors.

\section{References}

1. Fire A, Xu S, Montgomery MK, Kostas SA, Driver SE and Mello CC: Potent and specific genetic interference by double-stranded RNA in Caenorhabditis elegans. Nature 391: 806-811, 1998

2. Gandellini P, Folini M, Bandiera R, De Cesare M, Binda M, Veronese S, Daidone MG, Zunino $\mathrm{F}$ and Zaffaroni $\mathrm{N}$ : Down-regulation of human telomerase reverse transcriptase through specific activation of RNAi pathway quickly results in cancer cell growth impairment. Biochem Pharmacol 73: $1703-1714,2007$.

3. Harley CB, Futcher AB and Greider CW: Telomeres shorten during ageing of human fibroblasts. Nature 345: 458-460, 1990.

4. Livak KJ and Schmittgen TD: Analysis of relative gene expression data using real-time quantitative PCR and the 2(-Delta Delta C(T)) Method. Methods 25: 402-408, 2001.

5. Hastie ND, Dempster M, Dunlop MG, Thompson AM, Green DK and Allshire RC: Telomere reduction in human colorectal carcinoma and with ageing. Nature 346: 866-868, 1990.

6. McFadden N, Bailey D, Carrara G, Benson A, Chaudhry Y, Shortland A, Heeney J, Yarovinsky F, Simmonds P, Macdonald A and Goodfellow I: Norovirus regulation of the innate immune response and apoptosis occurs via the product of the alternative open reading frame 4. PLoS Pathog 7: e1002413, 2011.

7. Folini M, Brambilla C, Villa R, Gandellini P, Vignati S, Paduano F, Daidone MG and Zaffaroni N: Antisense oligonucleotide-mediated inhibition of hTERT, but not hTERC, induces rapid cell growth decline and apoptosis in the absence of telomere shortening in human prostate cancer cells. Eur J Cancer 41 624-634, 2005
8. Cong YS, Wright WE and Shay JW: Human telomerase and its regulation. Microbiol Mol Biol Rev 66: 407-425, 2002.

9. Blackburn EH, Greider CW and Szostak JW: Telomeres and telomerase: The path from maize, Tetrahymena and yeast to human cancer and aging. Nat Med 12: 1133-1138, 2006.

10. Zou L, Zhang P, Luo C and Tu Z: shRNA-targeted hTERT suppress cell proliferation of bladder cancer by inhibiting telomerase activity. Cancer Chemother Pharmacol 57: 328-334, 2006.

11. Gorbunova V, Seluanov A and Pereira-Smith OM: Expression of human telomerase (hTERT) does not prevent stress-induced senescence in normal human fibroblasts but protects the cells from stress-induced apoptosis and necrosis. J Biol Chem 277: 38540-38549, 2002.

12. Liu K, Hodes RJ and Weng Np: Cutting edge: Telomerase activation in human $\mathrm{T}$ lymphocytes does not require increase in telomerase reverse transcriptase (hTERT) protein but is associated with hTERT phosphorylation and nuclear translocation. J Immunol 166: 4826-4830, 2001.

13. O'Connor DS, Schechner JS, Adida C, Mesri M, Rothermel AL, Li F, Nath AK, Pober JS and Altieri DC: Control of apoptosis during angiogenesis by survivin expression in endothelial cells. Am J Pathol 156: 393-398, 2000.

14. Porter AG and Jänicke RU: Emerging roles of caspase-3 in apoptosis. Cell Death Differ 6: 99-104, 1999.

15. Dale-Nagle EA, Satriotomo I and Mitchell GS: Spinal vascular endothelial growth factor induces phrenic motor facilitation via extracellular signal-regulated kinase and Akt signaling. J Neurosci 31: 7682-7690, 2011.

16. Paterniti I, Esposito E, Mazzon E, Bramanti P and Cuzzocrea S: Evidence for the role of PI(3)-kinase-AKT-eNOS signalling pathway in secondary inflammatory process after spinal cord compression injury in mice. Eur J Neurosci 33: 1411-1420, 2011.

17. Gheysarzadeh A and Yazdanparast R: Inhibition of $\mathrm{H} 2 \mathrm{O} 2$-induced cell death through FOXO1 modulation by EUK-172 in SK-N-MC cells. Eur J Pharmacol 697: 47-52, 2012.

18. Park HS, Seong KM, Kim JY, Kim CS, Yang KH, Jin YW and Nam SY: Chronic low-dose radiation inhibits the cells death by cytotoxic high-dose radiation increasing the level of AKT and acinus proteins via NF- $\kappa \mathrm{B}$ activation. Int J Radiat Biol 89: 371-377, 2013.

19. Yang W, Ju JH, Lee KM, Nam K, Oh S and Shin I: Protein kinase B/Akt1 inhibits autophagy by down-regulating UVRAG expression. Exp Cell Res 319: 122-133, 2013.

20. Zeng $X$, Huang Z, Mao X, Wang J, Wu G and Qiao S: $\mathrm{N}$-carbamylglutamate enhances pregnancy outcome in rats through activation of the PI3K/PKB/mTOR signaling pathway. PLoS One 7: e41192, 2012.

21. Siraskar B, Völkl J, Ahmed MS, Hierlmeier M, Gu S, Schmid E, Leibrock C, Föller M, Lang UE and Lang F: Enhanced catecholamine release in mice expressing PKB/SGK-resistant GSK3. Pflugers Arch 462: 811-819, 2011.

22. Vichalkovski A, Gresko E, Hess D, Restuccia DF and Hemmings BA: PKB/AKT phosphorylation of the transcription factor Twist-1 at Ser42 inhibits p53 activity in response to DNA damage. Oncogene 29: 3554-3565, 2010. 